\title{
Putting the Sexy Back into Safer Sex: The Pleasure Project
}

\section{Wendy Knerr and Anne Philpott}

\author{
Putting the sexy back into safer sex ... \\ Because sex education is rarely sexy \\ And erotica is rarely safe.
}

\section{Sexual pleasure is a key component of sexual health}

As Ingham (2005) observes, a greater acceptance of positive sexual experience can hold benefits for public health outcomes. Other studies have found that denying the possibility of pleasure in sexual relations, especially for women, has a negative impact on active negotiation and therefore safer sex (Holland et al. 1992).

Sexual pleasure is beginning to be recognised as a determinant of sexual health in the reproductive health and human rights fields. For example, the World Health Organization (WHO) offers this working definition of sexual health:

\section{Sexual health is a state of physical, emotional, mental and social well-being in relation to sexuality; it is not merely the absence of disease, dysfunction or infirmity. Sexual health requires a positive and respectful approach to sexuality and sexual relationships, as well as the possibility of having pleasurable and safe sexual experiences, free of coercion, discrimination and violence ... [emphasis added]. ${ }^{1}$}

In 2005 at the 17th World Congress for Sexology, the World Association for Sexual Health declared that sexual pleasure and satisfaction are integral to well-being and require universal recognition and promotion. $^{2}$

It is widely recognised that pleasure can motivate unsafe sex. However, there has been limited exploration of the potential for pleasure to motivate safer sex, or the contexts in which it may work most effectively. The good news is a few health programmes and condom promotion campaigns are now being bold enough to include pleasure as a motivating factor, and show how greater sensitivity and sensuality can combine with condom use. An increase in the uptake of condoms and safer sex has followed. ${ }^{3}$

The Pleasure Project ${ }^{4}$ builds bridges between the pleasure/sex industry and the safer sex world by avoiding negativity, and by ensuring that erotic materials include examples of safer sex and that sexual health and training materials include pleasure as a key element. The Pleasure Project avoids negativity, promoting safer sex by focusing on some of the prime motivators for having sex: desire and pleasure. Evidence suggests that positive incentives provide the most effective way to get people to want to have safer sex, and the project works to provide sex education with the emphasis on 'good sex'. In this article, we give a brief account of some of the Pleasure Project's work and reflect on how a more sex-positive approach to safer sex can help promote greater sexual well-being.

\section{Pleasure propaganda for safer sex: a global mapping}

The Pleasure Project conducted a global mapping to identify projects/organisations worldwide that promote pleasure in HIV prevention and sexual health promotion, and include safer sex in sexually provocative media. ${ }^{5}$ This mapping was compiled through research that included postings on sexual health list-serves, personal communications at meetings and conferences, and a snowballing of contacts reached through initial contacts. Information was gathered by telephone, email and web research. The vast majority of information took the form of grey literature and personally recounted programme strategies. It resulted in a collection of more than 30 organisations, programmes, services, events and resources that creatively use sexual pleasure as a primary motivation for practising safer sex. 
The mapping exercise documented a rich diversity of initiatives in a variety of contexts, and showed how they had been able to successfully promote pleasure as a sexual health strategy. These included programmes which eroticise male and female condoms; sex-positive books for teenagers; work with churches to improve sex among married couples; safer sex erotica designed for HIV-positive people; and pleasure and harm-reduction counselling for sex workers. The following are some examples.

The Vida Positiva (Positive Living) programme in Mozambique, an initiative of the South African organisation Empowerment Concepts, ${ }^{6}$ promotes safer sex by tackling the boredom with their sex lives at home that was leading men to have sex outside marriage. Educators worked with community 'gatekeepers', including religious leaders from Christian churches, to promote pleasure-focused couples counselling. As we note in the Global Mapping report, 'this is one of the best examples there is of harnessing the power of pleasure, even within traditionally conservative environments like the church!' (The Pleasure Project 2004: 3).

- Sex in Queer Places, an initiative of the Australian Federation of AIDS Organisations, ${ }^{7}$ is an interactive videogame that enables viewers to follow a fictional gay man named Zac through a variety of sexual encounters in queer places such as a sauna and a dance party, making a series of sexual and drug-use choices along the way. As each choice is made, a dialogue box pops up with health promotion and harm minimisation information in it. Thousands of copies of the CD, along with an electronic booklet that takes a sexpositive approach to sex for HIV-positive men, have been distributed.

- Teaching the Kama Sutra is an initiative of the Institute for International Social Development (IISD) in Kolkata, a non-governmental organisation (NGO) working on HIV/AIDS awareness, prevention, treatment and rehabilitation. Supported by the West Bengal State government, the IISD's programme seeks answers to contemporary safer sex concerns in the ancient pleasure-focused practices of the Kama Sutra. Rajyashree Choudhuri, IISD's chief and architect of the project points out, 'Kama Sutra has many postures that can give men the highest pleasure without consummation and that is what prostitutes are being taught' (The Pleasure Project 2004: 7). Safer pleasures inspired by the Kama Sutra are also being promoted by other organisations in India, such as the Sonagachi Project in Kolkata, and the People's Health Organisation in Mumbai.

- Pleasure Helpline - TARSHI, a Delhi-based NGO and host to the Ford Foundation-funded South and South East Asia Resource Centre on Sexuality, operates a telephone helpline that provides information and discussion about sexual pleasure and sexual health, using sexual concerns and pleasure as an entry point for further discussions on health behaviour. Guided by the vision that 'all people have a right to sexual wellbeing and to a self-affirming and enjoyable sexuality' (The Pleasure Project 2004: 10), TARSHI also develops publications and conducts advocacy initiatives and public campaigns.

- 'Inside-Out' Training for Social Change: Ibis, a Namibia-based NGO, working on organisational development, local governance and popular participation, runs a regional HIV/AIDS programme. Staff and partners undergo an internal organisational training process as agents of change. This process involves enabling staff to explore their own realities and responsibilities, breaking through personal denial and helping to lift taboos that surround talking about sex and sexuality among colleagues and friends. Mixed workshops bring top management and cleaning staff together to explore intimate issues across boundaries of class and status. Global Mapping reports on one of the exercises that is used in these workshops, which involves two teams being asked to list as many parts of the body that they think can be used in a sexual way, and as many sexual positions as they can. Through laughter and fun, issues such as the variety of possibilities for sexual pleasuring emerge - making the point that much of what is pleasurable need not involve penetrative sex, and leading to discussions about the safety of a variety of non-penetrative sexual activities.

Eroticising Female Condoms: Anne Philpott has collated a variety of techniques for negotiating female condom use, which are based on promoting it as a tool for enhancing pleasure. She 
found that in Sri Lanka, sex workers could charge clients more money when they allowed them to insert the female condom, and in India women told partners that the condom only made a noise when the male partner was good in bed. As people found from experience that the outer ring of the condom stimulates the clitoris and the inner ring stimulates the end of the penis they were able to pass this on to others, turning female condoms from disease prevention tools into sex toys. ${ }^{9}$

- Marketing Lube for Pleasure: Population Services International in Cambodia use social marketing to promote sexual health products, and launched their water-based lubricant with a campaign that drew attention to its pleasure benefits. Packaging their lubricant together with a popular brand of condom, this campaign promoted the use of them together by emphasising the pleasure benefits. ${ }^{10}$

- Sexual Healing for Positive Women: Sue O'Sullivan's book for the International Community of Women Living with HIV and AIDS (ICW) combines erotica and messages about health for HIV-positive women. Its aim is to enable positive women to talk about sex after receiving a positive diagnosis, to affirm their sexuality and to ensure that they remain able to seek and enjoy sexual pleasures. ${ }^{11}$

The Global Mapping project revealed the extent to which these inspiring examples remain isolated in a field that continues to be dominated by conventional sexual health promotion strategies, which may pay little attention to the power of pleasure. The Pleasure Project went on to develop a series of 'Pleasure + Prevention' case studies. These case studies provide more detailed documentation of innovative practices. They demonstrate the value of taking a sex-positive approach to working with sexuality.

\section{Sexy sexual-health training}

As well as gathering and sharing examples of innovative practices promoted by organisations in different parts of the world, the Pleasure Project has been involved in the design and development of training materials.

The Pleasure Project worked with CARE International in Cambodia to design and run a sexy sexual health training programme, and to develop a toolkit of training materials. The course, which ran for three days
Figure 1 During the training, participants brainstormed about what pleases them sexually

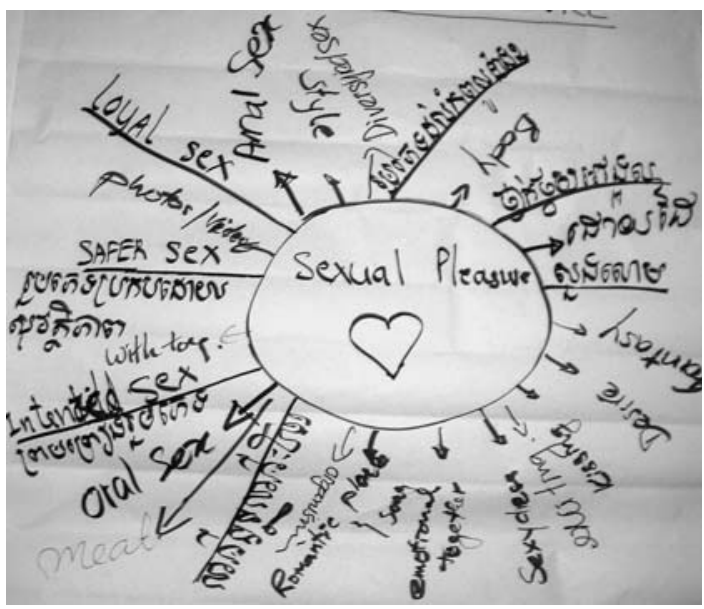

in December 2004, aimed to enable participants to become more comfortable talking about sex and pleasure so that they could be more effective in teaching others about safer sex.

The trainees were male and female sexual health trainers and peer educators who work with young people in two CARE projects: the 'Sewing a Healthy Future' (SHF) project which aims to develop local capacity to meet the needs of 50,000 garment factory workers, predominantly young single migrant women, for HIV/AIDS prevention and family planning information and services; and the 'Playing Safe' (PS) project which aims to contribute to improved reproductive and sexual health among 20,000 young urban men in Phnom Penh. Both projects use a peer education approach and participatory life skill trainings which cover basic reproductive health and HIV/AIDS prevention messages. SHF trainings, mainly targeted at young single working women, include a focus on negotiation skills within relationships and social support mechanisms to help them cope with city life. PS training includes addressing cultural stereotypes for young males, sexual coercion and violence, as well as promoting human rights and gender awareness.

A series of exercises were developed and piloted. Some of the most popular exercises include one that encourages people to discuss frankly their fears about sex, one that opens up discussion on sexual pleasure by beginning with a focus on the pleasure 
of eating and moving from there to find a language with which to talk about the pleasures of sex, and one that maps the 'places of pleasure' on the female and male body, and explore what feels good and what does not. Such exercises have proven to be popular and effective ways of breaking the silences that surround sex and sexual pleasure, and overcoming taboos, creating space for talking about how to make sex sexier as well as safer.

The Pleasure Project aimed to enhance these trainings by enabling trainers and educators to learn how to use pleasure as both an opening and an ongoing way to motivate safer sex.

At the beginning of the training, many participants felt shame and reluctance to discuss such issues. However by the end discussion was far more open, demonstrating that it is indeed possible to overcome such inhibitions. Two kinds of barriers - internal and external - were identified and strategies developed to overcome them. Internal barriers comprise participants' reluctance to discuss such issues and these relate very strongly to the shame associated with discussing sex publicly. External barriers relate to constraints perceived as coming from communities or from 'culture' more broadly. Participants admitted that people do indeed talk about sex in specific particular situations (among peers, for example). An extended discussion using the association between pleasure and food demonstrated the potential value of metaphor in situations where open discussion of sexual pleasure may not be possible.

Other potential barriers and restrictions were identified and addressed with varying degrees of success. One such barrier relates to gender and highlights the importance of working whenever possible in single-sex groups. The fact that there were far more female than male participants meant that this was not feasible and despite efforts to

\section{Notes}

1 WHO's working definition of sexual health: www.who.int/reproductive-health/gender/ sexual_health.html (accessed 23 January 2006).

2 World Association for Sexual Health: www.seksuologen-nederland.nl/html/Montreal\% 20Declaration\%20Sexual\%20Health\%20for\%20th e\%20Millennium.pdf Montreal Canada, 15 July 2005, XVII World Congress of Sexology (accessed 23 January 2006). safeguard against this, some male participants tended to dominate the discussions. A more equal gender balance among participants would make it possible for much of the training (particularly the more personally oriented activities), to be run in two separate but parallel groups, thereby encouraging female participants to speak more freely. $A$ male/female facilitator team can be useful in terms of demonstrating gender equality and modelling a frank discussion of sex and pleasure.

As a result of the training, sexual health trainers were able to better integrate discussion of pleasure within their work and address some of the barriers to free discussion that arise in a training situation, creating an environment that supports people to discuss intimate issues. It is clear from this experience that with support, sexual health trainers can be enabled to discuss sex and pleasure, even in what are perceived to be more conservative cultures. By the end of the training, almost without exception, participants expressed a commitment to extending this discussion into their own practice.

\section{Conclusion}

Safer sex and good sex are not mutually exclusive, yet most established educational programmes give this impression by using fear and the risk of disease to motivate their audience to practise safer sex. There is nothing revolutionary about taking a pleasurable approach to safer sex - all evidence suggests that positive incentives are far more effective change motivators than fear. What the Pleasure Project's work is showing is that taking a sex-positive, pleasure-focused approach to working with sex and sexuality can make an important difference to sexual health outcomes. The results of this kind of approach, however, go beyond improving sexual health, to enabling women and men to experience healthier, happier lives - a development goal in itself.

3 See 'Eroticizing the Female Condom, How to Increase Usage', Reference No 350823, ICASA African AIDS Conference, July 2003; 'Kamasutra' invites UN attraction, AIDS-INDIA (19 October 2003); BBC News Kama Sutra Guide to Safer Sex, 8 August 2003, http://news.bbc.co.uk/2/hi/ south_asia/3133947.stm; Marie Stopes International Mongolia, Project Sales Reports for Female Condom Programme 2003. 
4 The Pleasure Project: see www.the-pleasureproject.org for further information; see also Venis (2005).

5 The Global Mapping report outlines 27 initiatives, along with contact details of the organisations involved. This article draws substantially on this report. See www.the-pleasure-project.org/ Global\%20Mapping\%20of\%20Pleasure.pdf

6 Further information on Empowerment Concepts and their work can be found at www.empowermentconcepts.com

7 Australian Federation of AIDS Organisations: see www.afao.org.au for further information.

\section{References}

Holland, J., Ramazanoglu, C., Scott, S., Sharpe, S. and Thomson, R. (1992) 'Risk, Power and the Possibility of Pleasure: Young Women and Safer Sex', AIDS Care 4.3: 273-83

Ingham, Roger (2005) "'We Didn't Cover That at School": Education Against Pleasure or Education for Pleasure?', Sex Education 5.4: 375-88
8 More information on TARSHI can be found at www.tarshi.org

9 This is reported in more detail in an article in The Guardian, 'Whatever Happened to the Femidom', 23 August 2005: www.guardian.co.uk/g2/story/ $0,1554324,00 \cdot \mathrm{html}$

10 This experience has been documented in detail as the Pleasure Project 'Pleasure + Prevention' case study. See www.the-pleasure-project.org for further details.

11 For more information on ICW and the Sexual Healing resource, see www.icw.org

The Pleasure Project (2004) Global Mapping of Pleasure, www.the-pleasure-project.org Venis, Sarah (2005) 'Lunch with the Lancet: Anne Philpott', The Lancet 365 (9459): 565 